\title{
MONO-ENERGETIC BEAMS FROM LASER PLASMA INTERACTIONS*
}

\author{
C.G.R. Geddes ${ }^{\sharp \S}$, E. Esarey, W.P. Leemans ${ }^{+}$, C.B. Schroeder, Cs. Toth, J Van Tilborg ${ }^{\dagger}$, LBNL, \\ Berkeley, California
}

John R. Cary, CIPS and Tech-X, Boulder, Colorado

David L. Bruhwiler, Chet Nieter (Tech-X, Boulder, Colorado)

\begin{abstract}
A laser driven wakefield accelerator has been tuned to produce high energy electron bunches with low emittance and energy spread by extending the interaction length using a plasma channel. Wakefield accelerators support gradients thousands of times those achievable in RF accelerators, but short acceleration distance, limited by diffraction, has resulted in low energy beams with $100 \%$ electron energy spread. In the present experiments on the L'OASIS laser, the relativistically intense drive pulse was guided over 10 diffraction ranges by a plasma channel. At a drive pulse power of $9 \mathrm{TW}$, electrons were trapped from the plasma and beams of percent energy spread containing $>200 \mathrm{pC}$ charge above $80 \mathrm{MeV}$ and with normalized emittance estimated at $<2 \pi-\mathrm{mm}$-mrad were produced. Data and simulations (VORPAL code) show the high quality bunch was formed when beam loading turned off injection after initial trapping, and when the particles were extracted as they dephased from the wake. Up to $4 \mathrm{TW}$ was guided without trapping, potentially providing a platform for controlled injection. The plasma channel technique forms the basis of a new class of accelerators, with high gradients and high beam quality.
\end{abstract}

\section{INTRODUCTION}

A promising candidate for the next generation of compact high-energy electron sources is the laser wakefield accelerator (LFWA), which has demonstrated accelerating gradients thousands of times those obtained in conventional accelerators using the electric field of a plasma wave (the wakefield) driven by an intense laser[13], indicating the potential for more compact accelerators. Acceleration distance and hence electron beam energy and quality has been limited however by the difficulty of retaining high laser intensity over a long distance of propagation in the plasma, resulting in poor quality electron bunches with $100 \%$ energy spread and an exponentially small fraction of electrons at high energy[13]. Laser power in past experiments was above the critical power for self-focusing and the laser pulse length exceeded the plasma wavelength. In this self modulated regime, some self-guiding of the laser pulse occurs due to relativistic modification of the plasma refractive index, but the laser pulse is highly unstable [4, 5], limiting propagation length to little more than a diffraction range $Z_{R}[6]$. The best results had therefore been obtained by increasing spot size to increase $Z_{R}$, requiring ever greater laser power, but this approach had still been limited to distances of a few hundred micron[6]. To circumvent this limit and to realize the potential of laser accelerators, the laser pulse should be guided at relativistic intensities, and its propagation controlled over distances of several $\mathrm{mm}$ or greater [7].

\section{EXPERIMENT}

We report the first guiding of relativistic laser intensities over many diffraction ranges by a plasma channel, and the resulting production of electron bunches of few percent energy spread, hundreds of picoCoulombs of charge, and milliradian divergence at energies above $80 \mathrm{MeV}$. The high acceleration gradients of previous laser accelerator experiments are retained over longer distances, and the beam quality is comparable to state of the art RF accelerators. Experiments and supporting simulations indicate that the high quality bunches are formed from self trapped electrons when beam loading turns off self trapping after the loading of an initial bunch, and when the bunch is accelerated to the dephasing length over which trapped electrons outrun the wake.

Guiding at high intensities to produce a channel guided accelerator required controlling for both diffraction and plasma effects. Previous experiments demonstrated guiding for input pulse intensities at up to $2 \times 10^{17} \mathrm{~W} / \mathrm{cm}^{2}[8-11]$, where a parabolic transverse plasma density profile can be matched to guide the low intensity pulse[12]. At high intensities, relativistic self guiding occurs when the quiver motion of the electrons causes their mass to increase, changing the refractive index. This occurs in regimes of interest to wakefield acceleration and provides some self guiding, but is unstable $[4,5]$. The channel must balance both diffraction and instabilities to allow long propagation distance at high intensity.

\footnotetext{
* Supported by U.S. Dept. of Energy contracts DE-AC03-76SF00098, DE-FG03-95ER40926, DE-FG02-01ER41178, DE-FG0203ER83857, SciDAC, and NSF 0113907. C. Geddes is also supported by the Hertz foundation.

"corgeddes@lbl.gov

$\S$ also at University of California, Berkeley

${ }^{+}$http://loasis.lbl.gov

${ }^{\dagger}$ also at Technische Universiteit Eindhoven
} 

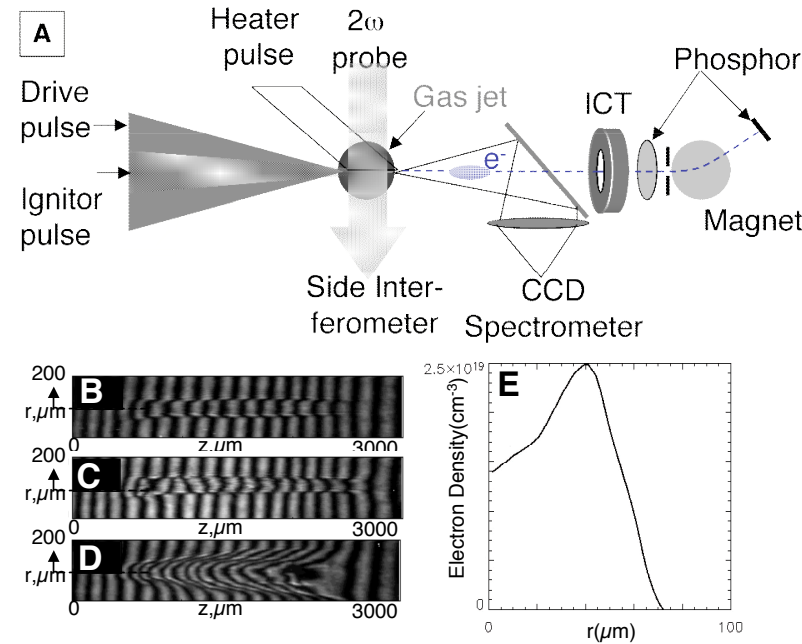

Figure 1. (a) Experimental setup showing the gas jet with laser beams and diagnostics. (b-d) Side interferometer images show the channel structure and laser propagation. 500ps after formation, at the time of drive pulse arrival, a channel was formed (a) and the density profile was obtained by Abel inversion (e). Image of the drive beam at $4 \mathrm{TW}$ propagating in this channel (c) is similar to (b) indicating good confinement, while the unguided beam (d) diffracts rapidly.

The multi arm l'OASIS Ti:Sapphire laser[13, 14], operating at $800 \mathrm{~nm}$ with chirped pulse amplification, was used to form the guiding channel using a variation of the ignitor-heater method[9] and to drive the plasma wake (Figure $1 \mathrm{~A}$ ). A plasma was ionized by an ignitor pulse $(15 \mathrm{~mJ}, 60 \mathrm{fs})$ from a $2.5 \mathrm{~mm}$ long supersonic $\mathrm{H}_{2}$ gas jet with an atomic density of $3-4 \times 10^{19} \mathrm{~cm}^{-3}$, then heated to 10 's of eV by a heater pulse (using $\sim 50 \mathrm{~mJ}$ from a $150 \mathrm{~mJ}$, $250 \mathrm{ps}$ beam). Hydrodynamic expansion of the plasma drove a shock in the surrounding neutral gas, forming a channel with a nearly parabolic transverse density distribution [8]. This channel was used to guide the relativistically intense drive pulse focused at its edge. In order to drive an intense wake capable of trapping and accelerating electrons, the drive pulse $(500 \mathrm{~mJ}, 55 \mathrm{fs})$ was focused to a spot of 7-8.5 $\mu \mathrm{m}$ FWHM to reach intensities up to $1.1 \mathrm{E} 19 \mathrm{~W} / \mathrm{cm}^{2}$, . This gave $\mathrm{Z}_{\mathrm{R}} \sim 200 \mu \mathrm{m}$ so that the channel was $>10 Z_{R}$ long. Propagation of the laser was monitored with a side interferometer, mode imager CCD, and transmitted light spectrometer. Electrons accelerated by the plasma wake of the drive beam were analyzed using an integrating current transformer (ICT), a phosphor screen, and a magnetic spectrometer.

\section{LASER GUIDING}

By adjusting the timing of the beams, the channel profile has been adjusted to guide the drive pulse at various powers and to compensate for the presence of self guiding[15]. With the channel tuned to match the low power guiding condition, aberration free guiding of low power pulses $(0.5 \mathrm{TW}<$ Pcrit $=$ critical power for self guiding) was obtained with transmission efficiency of $\sim 50 \%$, but pulses above $\mathrm{P}_{\text {crit }}$ were aberrated, displaying enlarged output spot size and lowered intensity. Retuning the channel to slightly flatten the density profile allowed compensation for the presence of self guiding, and powers up to $4 \mathrm{TW}\left(7 \mathrm{E} 18 \mathrm{~W} / \mathrm{cm}^{2}\right)$ were guided without aberration. The channel interferogram for this tune at the time of main pulse arrival is shown in Figure 1b. Propagation of the main beam in the channel does not change the image (Figure 1c), indicating that the laser is well confined to the channel, as leakage outside the channel would ionize additional gas. The unguided beam diffracts rapidly, demonstrating that self guiding alone is insufficient to control mode propagation over this distance (Figure 1d). The plasma density profile of the channel (Figure 1e) shows a nearly parabolic transverse dependence, with a rise in density over the spot diameter $\sim 40 \%$ less than the low power matching condition reflecting the adjustment made to compensate for self guiding.

Figure 2 shows the mode images of the laser spot propagation at $4 \mathrm{TW}\left(7 \mu \mathrm{m}\right.$ input spot, $\left.7 \mathrm{E} 18 \mathrm{~W} / \mathrm{cm}^{2}\right)$. With the channel on, the output spot (b) matches the input (a). The mode imager resolution is restricted by $\mathrm{f} / \#$ constraints in the target chamber so that it yields a $12 \mu \mathrm{m}$ FWHM spot size for both input and output. Deconvolution of instrument response[15] indicated that the output is consistent with a spot within $1 \mu \mathrm{m}$ of the 7 $\mu \mathrm{m}$ input spot size, giving output intensity $\sim 2.5 \times 10^{18}$ $\mathrm{W} / \mathrm{cm}^{2}$ (lower limit $1 \times 10^{18}$ set by the $12 \mu \mathrm{m}$ mode imager observation). The vacuum output displays diffraction (c), indicating the effectiveness of the guide, and with the gas jet on but the channel off (d) diffraction is increased by ionization effects[16, 17], showing that self guiding alone is insufficient to efficiently guide the beam.

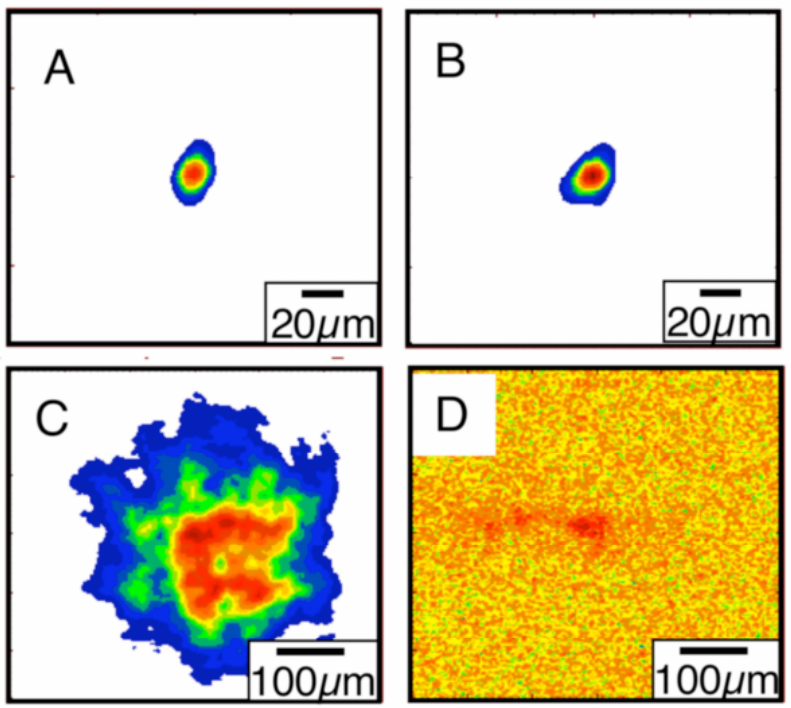

FIGURE 2. Mode images of laser propagation at $4 \mathrm{TW}$. The output image with the guide on (b) is indistinguishable from the input (a) indicating unaberrated propagation at twice $\mathrm{P}_{\text {crit }}$. Unguided images show diffraction in vacuum (c) and ionization enhanced diffraction with the gas jet on (d). 
Transmission at 4 TW was $35 \%$, a reduction of one third from the low power case, indicating that substantial power was deposited in plasma waves. The depletion observed is consistent with particle in cell simulations run with the experimental parameters (below), which indicate that a plasma wave averaging $2-300 \mathrm{GV} / \mathrm{m}$ is excited in the last $0.5 \mathrm{~mm}$ of guide length. No electrons are self trapped at $4 \mathrm{TW}$, making this an attractive structure for controlled injection experiments[18, 19]. Quality and stability of laser accelerated electron bunches may be greatly increased by controlling injection rather than relying on instabilities such as self modulation, but such experiments have until now been hampered by lack of a long scale length high gradient structure such as the channel described here can provide. Experiments on colliding pulse injection[19] are now under way in this geometry.

The transmitted spectrum of the laser pulse was also analyzed as a diagnostic of channel confinement and plasma wave excitation. Without the guiding channel, the spectrum showed severe blue shifting, which occurs when the laser ionizes the gas it is passing through[20]. This feature was nearly eliminated for the channeled beam, indicating that the laser was well confined to the guiding channel. The spectrum was close to undistorted with transmission of the $800 \mathrm{~nm}$ feature near $30 \%$ at $4 \mathrm{TW}$, close to the transmission observed on the mode imager. A red shifted shoulder also appeared in the channeled case, increasing in relative amplitude with increasing laser power, consistent with depletion of the laser into plasma waves.

\section{CHANNELED WAKEFIELD ACCELERATION}

At guided drive pulse powers above $4 \mathrm{TW}$, electrons were trapped and accelerated, verifying that an intense plasma wake was driven in the channel. At 9 TW, optimal performance was found in a channel with an axial density of $1.9 \times 10^{19} \mathrm{~cm}^{-3}$ and with a parabolic profile with $40 \%$ less rise in density over a spot diameter than the low power matched case. The laser was well confined to the channel at this power but some aberration was always present, with output mode sizes near $24 \mu \mathrm{m}$ FWHM, likely due to the strong self guiding present here. The drive laser pulse was a factor of two longer than the linear plasma period, i.e. in the self-modulated regime. This regime was chosen to allow comparison to unchannelled experiments, and also because the slower phase velocity of the wake at high plasma density allowed trapping of background plasma electrons yielding high charge electron beams without a separate injector.

The channel guided accelerator produced high charge electron beams with low energy spread at high energy, and with low divergence[21]. Figure 3 a shows a bunch of $2 \times 10^{9}$ electrons within an energy spread of $\pm 2 \%$ centered at $86 \mathrm{MeV}$. Due to pointing fluctuations which change the incoupling of the drive beam to the guide, this feature varied shot to shot, and bunches with $3 \times 10^{9}$ electrons at
$78 \mathrm{MeV}$ were observed, as well as $1 \times 10^{9}$ electrons at energies up to $150-170 \mathrm{MeV}$. The charge in the bunches was calibrated using the ICT and radionuclide activation measurements, both of which were consistent. Total charge in the electron beam was near $2 \times 10^{10}$ electrons; the low energy portion can be separated using a bend magnet, leaving a high energy high quality bunch. The divergence of the bunches near
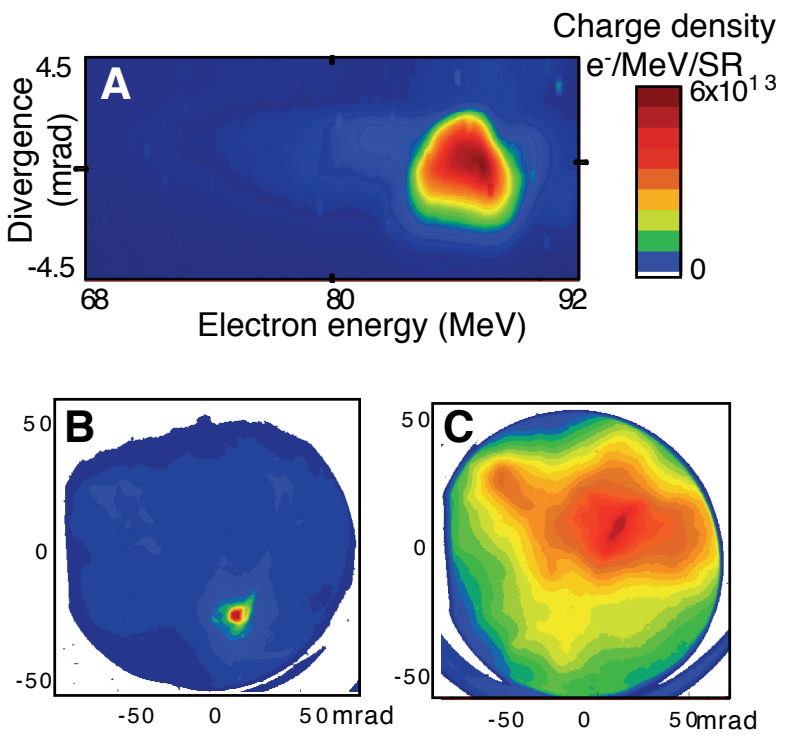

FIGURE 3. Electron bunches. The electron energy spectrum of the channeled accelerator (a) shows the appearance of monoenergetic features, here with 3E9 electrons in a bunch with energy spread of 4\% FWHM at $78 \mathrm{MeV}$. Divergence was near $3 \mathrm{mrad}$ FWHM for this bunch (a), and $6 \mathrm{mrad}$ for the whole beam (b). The unguided accelerator in the same gas jet by contrast shows a nearly smooth exponenetial spectrum with a few $\mathrm{MeV}$ temperature (not shown), and much wider divergence (c).

$80 \mathrm{MeV}$ was $3 \mathrm{mrad}$. This is half the divergence of the full beam observed before the magnet (Figure 3b), consistent with other experiments which have shown that the high energy portion of the beam is better collimated[2]. The normalized geometric emittance obtained from assuming the bunch comes from a source the laser spot size is $1-2 \pi-\mathrm{mm}$-mrad, competitive with state of the art radiofrequency facilities.

The accelerator was operated in the same gas jet without the guiding channel. Density was separately optimized for the unchanneled accelerator and best performance was at $\mathrm{n}_{\mathrm{e}}=4 \times 10^{19} \mathrm{~cm}^{-3}$. This produced an exponential energy distribution with a $2.6 \mathrm{MeV}$ temperature below $10 \mathrm{MeV}$ and an $8 \mathrm{MeV}$ temperature above $10 \mathrm{MeV}$. No electrons were observed (detection threshold $\sim 10^{7}$ electrons on the magnetic spectrometer phosphor) above $40 \mathrm{MeV}$. The bulk of the distribution was smooth, with occasional structure in the tail of the distribution containing $<2 \%$ of the charge. The beam divergence (Figure 3c) was much larger than the channeled case. No difference was observed between 
operation in a neutral gas jet and a pre-ionized (but not channeled) plasma, indicating that channeling and not ionization is the important effect.

\section{SIMULATIONS}

Two dimensional particle in cell simulations performed with parameters close to the experiment using the code VORPAL[22](developed at U. of Colorado/Tech X) show formation of bunches similar to those observed in the channeled accelerator. If laser pulse strength was just above that required to self trap electrons, loading of the wake[23] by the initial electron bunch trapped suppressed further injection. This lead to a bunch of electrons isolated in phase space (Figure 4 a). If this bunch was accelerated until it dephased from the wake, the leading edge of the bunch was decelerated while the tail was accelerated, concentrating the particles in energy and forming a low energy spread bunch at the dephasing length (Figure 4b). Matching accelerator length to the dephasing length for the jet length and $Z_{R}$ used required a guiding channel.
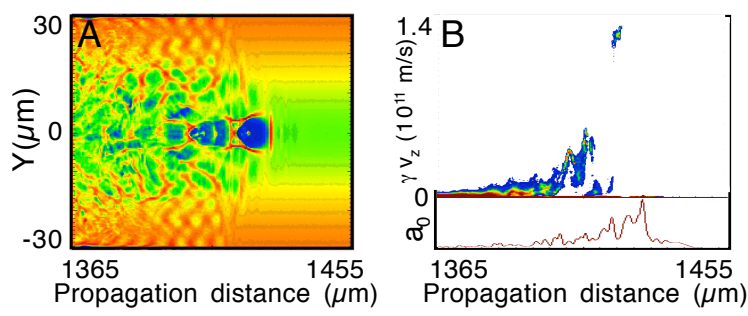

FIGURE 4. Particle in cell simulations show that a high quality bunch is formed when beam loading and pulse evolution turn off injection after the loading of an initial bunch, resulting in a bunch isolated in phase space. This is visible in the density contour (a), where the wake amplitude is lowered following the bunches visible in the first two buckets. These particles are then concentrated in energy at the dephasing length (b) forming a low energy spread bunch. The laser pulse envelope is shown at the bottom of pane (b), showing self modulation into subpulses at the plasma period.

Particles were tracked through the simulation to provide additional information on the particle trajectories (Figure 5). The particles are colored by injection order, with red particles being those injected first (at the shortest propagation distance), and blue last. The particle acceleration trajectories, Fig. 5A, verify that bunching in energy occurs when the front of the bunch (first injected particles) decelerates, while electrons at the tail (injected later) continue to accelerate. The transverse origin and subsequent behavior of the particles are shown in Figure 5B, indicating that the particles are injected from a radius of approximately $5 \mu \mathrm{m}$, rather than from on axis. This reflects the strong ponderomotive blowout by the laser pulse which excludes on axis electrons. A similar effect has also been seen in[24], though in much narrower channels where there was also interaction with the channel-vacuum interface. Formation of quasi- monoenergetic structures was previously observed in [25] for at much higher laser amplitudes than those here.
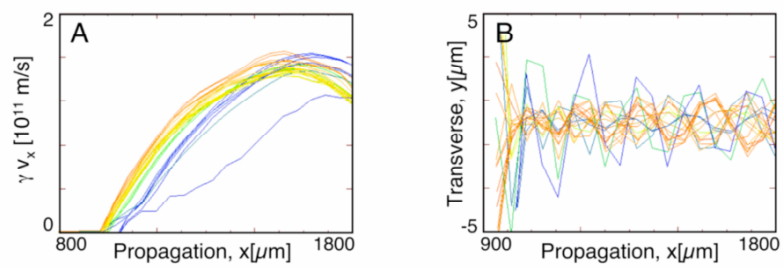

FIGURE 5. Tracks of representative particles in momentum vs. propagation distance (A) and transverse coordinate vs. propagation distance (B).

\section{DEPHASING EXPERIMENTS}

To evaluate the impact of dephasing on the accelerator experimentally, gas jets of variable length and density were used without channeling[26]. To meet the dephasing condition without channeling, a short gas jet can be used at the cost of reduced final energy, or self guiding can be used, though this is less stable than use of a channel. PIC simulations were used to evaluate dephasing length, since analytic estimates are not valid in this regime. With the available gas jet lengths and laser $Z_{R}$, it was possible to test at and after dephasing at a density of $4 \times 10^{19} \mathrm{~cm}^{-3}$, as well as before and after dephasing at a density of 2 $\mathrm{x} 10^{19} \mathrm{~cm}^{-3}$. The electron beam spectra showed that extraction of the beam before dephasing (600 $\mu \mathrm{m}$ plasma at $2 \times 10^{19} \mathrm{~cm}^{-3}$ ) yielded low energies, and a smooth spectrum. Extraction after dephasing $\left(4 \mathrm{~mm}\right.$ at $2 \times 10^{19} \mathrm{~cm}^{-3}$, or $2 \mathrm{~mm}$ at $4 \times 10^{19} \mathrm{~cm}^{-3}$ ) produced high energies with some structure in the distribution. The highest energies for a given density, as well as the most monoenergetic features in the spectrum, were obtained when the beam was extracted near the dephasing length $\left(600 \mu \mathrm{m}\right.$ at $4 \times 10^{19} \mathrm{~cm}^{-}$ $\left.{ }^{3}\right)$. This demonstrates the importance of tuning the accelerator to the dephasing length. Even with such optmization however, the unchanneled accelerator produced less stable, lower quality beams than the channeled accelerator, indicating the advantage of controlling and extending laser propagation length using the channel.

Consistent with the explanation presented here, monoenergetic beams were observed in experiments at other laboratories using a large laser spot size to increase $\mathrm{Z}_{\mathrm{R}}$ and hence the propagation distance of the laser [27, 28]. Like unchanneled experiments herein, this produced less accelerated charge and electron bunch energy per laser power because the large spot size reduced laser intensity, and hence wake amplitude and trapping.

\section{CONCLUSIONS}

Experiments have demonstrated guiding of relativistically intense laser pulses over many $Z_{R}$ in plasmas, and tailoring of the plasma profile to provide guiding without detectable aberration up to twice the relativistic self guiding threshold[15]. Input intensities near $10^{19} \mathrm{~W} / \mathrm{cm}^{2}$ have been guided without self injection of electrons. Increasing density and intensity produced 
self trapped electron beams of percent energy spread with several $10^{9}$ electrons and with emittance comparable to state of the art radio frequency accelerators[21]. This offers the possibility of new classes of experiments on laser driven accelerators and indicates that development of high energy high quality beams is feasible using this method, benefiting accelerator applications. Experiments and simulations indicate that these beams are formed by a combination of beam loading and dephasing[26]. Controlled injection using the colliding pulse method[19] will be tried in this structure, which may further stabilize and improve the bunch quality.

\section{ACKNOWLEDGEMENTS}

Work supported by the U.S. Dept. of Energy contracts DE-AC03-76SF00098, DE-FG03-95ER40926, DE-FG02-

\section{REFERENCES}

[1] A. Modena, Z. Najmudin, A. E. Dangor, C. E. Clayton, K. A. Marsh, C. Joshi, V. Malka, C. B. Darrow, C. Danson, D. Neely, and F. N. Walsh, Nature 377 (1995) 606.

[2] V. Malka, S. Fritzler, E. Lefebvre, M. M. Aleonard, F. Burgy, J. P. Chambaret, J. F. Chemin, K. Krushelnick, G. Malka, S. P. D. Mangles, Z. Najmudin, M. Pittman, J. P. Rousseau, J. N. Scheurer, B. Walton, and A. E. Dangor, Science 298 (2002) 1596.

[3] W. P. Leemans, P. Catravas, E. Esarey, C. G. R. Geddes, C. Toth, R. Trines, C. B. Schroeder, B. A. Shadwick, J. van Tilborg, and J. Faure, Physical Review Letters 8917 (2002) 4802.

[4] E. Esarey, J. Krall, and P. Sprangle, Physical Review Letters 72 (1994) 2887.

[5] E. Esarey, P. Sprangle, J. Krall, and A. Ting, IEEE Journal of Quantum Electronics 33 (1997) 1879.

[6] Z. Najmudin, K. Krushelnick, E. L. Clark, S. P. D. Mangles, B. Walton, A. E. Dangor, S. Fritzler, V. Malka, E. Lefebvre, D. Gordon, F. S. Tsung, and C. Joshi, Physics of Plasmas 10 (2003) 2071.

[7] E. Esarey, P. Sprangle, J. Krall, and A. Ting, IEEE Transactions on Plasma Science 24 (1996) 252.

[8] C. G. Durfee and H. M. Milchberg, Physical Review Letters 71 (1993) 2409.

[9] P. Volfbeyn, E. Esarey, and W. P. Leemans, Physics of Plasmas 6 (1999) 2269.

[10] K. Y. Kim, I. Alexeev, J. Fan, E. Parra, and H. M. Milchberg, in AIP Conf. Proc., 647, 646 (2002).

[11] E. W. Gaul, S. P. Le Blanc, A. R. Rundquist, R. Zgadzaj, H. Langhoff, and M. C. Downer, Applied Physics Letters 77 (2000) 4112.

[12] P. Sprangle, E. Esarey, J. Krall, and G. Joyce, Physical Review Letters 69 (1992) 2200.

[13] C. Toth, J. van Tilborg, C. G. R. Geddes, G. Fubiani, C. B. Schroeder, E. Esarey, J. Faure, G. Dugan, and W. P. Leemans, SPIE Proceedings 5448 (2004)
01ER41178, DE-FG02-03ER83857, NSF grant 0113907, and D.O.E. Office of Advanced Scientific Computing Research under the Scientific Discovery through Advanced Computing program. This research used resources of the National Energy Research Scientific Computing Center, supported by D.O.E contract DEAC03-76SF00098. C.Geddes is also supported by the Hertz foundation, and acknowledges his faculty advisor Jonathan Wurtele, at UC Berkeley. We appreciate contributions by Gerry Dugan, Jerome Faure, Gwenael Fubiani, Bob Nagler, Kei Nakamura, Ned Saleh, Brad Shadwick, Leon Archambault, Michael Dickinson, Scott Dimaggio, Don Syversrud, Joe Wallig, and Nathan Ybarrolaza.

[14] W. P. Leemans, P. Volfbeyn, K. Z. Guo, S. Chattopadhyay, C. B. Schroeder, B. A. Shadwick, P. B. Lee, J. S. Wurtele, and E. Esarey, Physics of Plasmas 5 (1998) 1615.

[15] C. G. R. Geddes, C. Toth, J. van Tilborg, E. Esarey, C. B. Schroeder, and W. P. Leemans, Submitted to Phys. Rev. Lett., 2004.

[16] R. Rankin, C. E. Capjack, N. H. Burnett, and P. B. Corkum, Optics Letters 16 (1991) 835.

[17] W. P. Leemans, C. E. Clayton, W. B. Mori, K. A. Marsh, P. K. Kaw, A. Dyson, C. Joshi, and J. M. Wallace, Physical Review A. Atomic, Molecular, and Optical Physics 46 (1992) 1091.

[18] D. Umstadter, J. K. Kim, and E. Dodd, Physical Review Letters 76 (1996) 2073.

[19] E. Esarey, R. F. Hubbard, W. P. Leemans, A. Ting, and P. Sprangle, Physical Review Letters 79 (1997) 2682.

[20] E. Esarey, G. Joyce, and P. Sprangle, Physical Review E. Statistical Physics, Plasmas, Fluids, \& Related Interdisciplinary Topics 44 (1991) 3908.

[21] C. G. R. Geddes, C. Toth, J. van Tilborg, E. Esarey, C. B. Schroeder, D. Bruhwiler, C. Nieter, J.

Cary, and W. P. Leemans, Nature 431 (2004) 538.

[22] C. Nieter and J. R. Cary, Journal of Computational Physics 196 (2004) 448.

[23] T. Katsouleas, S. Wilks, S. Chen, J. M. Dawson, and J. J. Su, Particle Accelerators 22 (1987) 81.

[24] F. S. Tsung, N. Ritesh, W. B. Mori, C. Joshi, R. A. Fonseca, and L. O. Silva, Physical Review Letters 93 (2004) 1.

[25] A. Pukhov and J. Meyer-Ter-Vehn, Applied Physics B Lasers \& Optics (2002) 4.

[26] C. G. R. Geddes, C. Toth, J. van Tilborg, E. Esarey, C. B. Schroeder, D. Bruhwiler, J. Cary, and W. P. Leemans, Submitted to Phys. Plasmas., 2004.

[27] J. Faure, Y. Glinec, A. Pukhov, S. Kisetev, S. Gordienko, E. Lefebvre, J. P. Rousseau, F. Burgy, and V. Malka, Nature 431 (2004) 541.

[28] S. P. D. Mangles, C. D. Murphy, Z. Najmudin, A. G. R. Thomas, J. L. Collier, A. E. Dangor, E. J. Divall, P. S. Foster, J. G. Gallacher, C. J. Hooker, D. A. Jaroszynski, A. J. Langley, W. B. Mori, P. A. 
Norreys, F. S. Tsung, R. Viskup, B. R. Walton, and K.

Krushelnick, Nature 431 (2004) 535. 